\title{
ON HYPER-REGULARITY AND UNIMODULARITY OF ORE POLYNOMIAL MATRICES
}

\author{
KLEMENS FRITZSCHE $^{a, *}$, KLAUS RÖBENACK ${ }^{a}$ \\ ${ }^{a}$ Institute of Control Theory, Faculty of Electrical and Computer Engineering \\ Dresden University of Technology, D-01062 Dresden, Germany \\ e-mail: \{klemens.fritzsche, klaus.roebenack\}@tu-dresden.de
}

\begin{abstract}
We investigate Ore polynomial matrices, i. e., matrices with polynomial entries in $\mathrm{d} / \mathrm{d} t$ whose coefficients are meromorphic functions in $t$ and as such constitute a non-commutative ring. In particular, we study the properties of hyper-regularity and unimodularity of such matrices and derive conditions which make it possible to efficiently check for these characteristics. In addition, this approach enables computation of hyper-regular left and right and unimodular inverses.
\end{abstract}

Keywords: Ore polynomial matrices, hyper-regularity, unimodularity, meromorphic functions, differential operator, noncommutativity, algorithm, hyper-regular inverse.

\section{Introduction}

Matrices with entries in differential operators play a key role in solving Serre's conjecture (Cluzeau and Quadrat, 2013; Fabianska and Quadrat, 2007; Lam, 1978; Logar and Sturmfels, 1992; Youla and Pickel, 1984) and thus are of interest in pure mathematics. Their occurence in applications such as control theory underlines this interest as well and motivates our work (see, e.g., the works of Franke and Röbenack (2013), Fritzsche et al. (2016), Lévine (2011), Middeke (2011), Newman (1972) or Zhou and Labahn (2014) and the references therein). In this paper, we investigate polynomial matrices with meromorphic entries in the differential operator $\frac{\mathrm{d}}{\mathrm{d} t}$ which leads to non-commutative operations. To show hyper-regularity or unimodularity, the so-called Smith normal form can be used. However, the computation is rather costly and thus not very practical. Instead, methods based on row and column reduction have been developed by Beckermann et al. (2006) as well as Antritter et al. (2014), Antritter and Middeke (2011) or Verhoeven (2016), with the last referencing a Maple toolbox. These methods also describe how to compute hyper-regular and unimodular inverses. While the row-reduced form of a matrix is directly related to the Popov normal form (Davies et al., 2008; Antritter et al., 2014), row reduction can be viewed as a special case of Gröbner basis

* Corresponding author computation (Middeke, 2011).

Instead of dealing with non-commutativity, in this contribution we will focus on the solvability of corresponding systems of linear equations and derive rank conditions that allow us to prove hyper-regularity and unimodularity, respectively. To this end, we will introduce operators which allow a reformulation of the problem as a system of linear equations and thus conclusions about solvability as well as solutions. These operators can easily be implemented in computer algebra systems.

With a free and open source 1 Python toolbox (Fritzsche, 2018) based on SymPy (Meurer et al., 2017) the presented examples can be reproduced.

\section{Preliminaries}

Let $\mathfrak{K}$ be the field of meromorphic functions in $t$. $\mathrm{GL}_{n}(\mathfrak{K})$ is the set of $n \times n$ matrices over $\mathfrak{K}$ which are regular almost everywhere, 2 while $\operatorname{Sym}(n)$ is the set of all permutations of degree $n$. $\quad$ By $\operatorname{row}(\mathbf{A}, \mathbf{B})$ we will denote $\left(\begin{array}{c}\mathbf{A} \\ \mathbf{B}\end{array}\right)$ for matrices $\mathbf{A}$ and $\mathbf{B}$. For the sake of readability, we will symbolize the differential operator $\frac{\mathrm{d}}{\mathrm{d} t}$ by $\lambda$.

The multiplication of two elements $a$ and $b$ of the Ore polynomial ring $\mathfrak{K}[\lambda]$ is non-commutative and determined

\footnotetext{
${ }^{1}$ GNU General Public License, Version 3.

2 The rank of a matrix in $\mathfrak{K}$ depends on $t$ and thus may have singularities, which will be ignored here. For example, $\left(\begin{array}{cc}t & 0 \\ 0 & t\end{array}\right) \in \mathrm{GL}_{2}(\mathfrak{K})$.
} 
by the rule

$$
\forall a \in \mathfrak{K}: \lambda a=\dot{a}+a \lambda,
$$

and for applying the operator $g$ times by

$$
\forall a \in \mathfrak{K}, g \in \mathbb{N}: \lambda^{g} a=\sum_{i=0}^{g}\left(\begin{array}{l}
g \\
i
\end{array}\right) a^{(g-i)} \lambda^{i} .
$$

Similarly, $\lambda$ can be applied from the right by the rule

$$
\forall a \in \mathfrak{K}: a \lambda=\lambda a-\dot{a},
$$

which generalizes to

$$
\forall a \in \mathfrak{K}, g \in \mathbb{N}: a \lambda^{g}=\sum_{i=0}^{g}(-1)^{i}\left(\begin{array}{l}
g \\
i
\end{array}\right) \lambda^{g-i} a^{(i)} .
$$

Definition 1. Let $\mathbf{A} \in \mathfrak{K}^{m \times n}$. A matrix $\mathbf{A}^{+R} \in \mathfrak{K}^{n \times m}$ is called a right pseudo-inverse of $\mathbf{A}$ if $\mathbf{A} \mathbf{A}^{+R}=\mathbf{I}_{m}$ holds. In the same manner, $\mathbf{A}^{+\mathrm{L}} \in \mathfrak{K}^{n \times m}$ is called a left pseudoinverse of $\mathbf{A}$ if $\mathbf{A}^{+\mathrm{L}} \mathbf{A}=\mathbf{I}_{n}$ holds.

Remark 1. Occasionally, (general) pseudo-inverses are defined such that $\mathbf{A} \mathbf{A}^{+} \mathbf{A}=\mathbf{A}$ and $\mathbf{A}^{+} \mathbf{A} \mathbf{A}^{+}=$ $\mathbf{A}^{+}$hold, which ensures pseudo-invertibility of (some) singular matrices (see the works of Ben-Israel and Greville (2003), Bose and Mitra (1978), Boullion and Odell (1971), Campbell and Meyer (2008) or Röbenack and Reinschke (2011) in the context of dynamical systems). In addition to the above conditions, the Moore-Penrose pseudo-inverse satisfies $\left(\mathbf{A}^{+} \mathbf{A}\right)^{\top}=$ $\mathbf{A}^{+} \mathbf{A}$ and $\left(\mathbf{A} \mathbf{A}^{+}\right)^{\top}=\mathbf{A} \mathbf{A}^{+}$, and although it can be used here, we can mostly be more permissive for the purpose of this paper.

Remark 2. Dealing with symbolic entries, pseudo-inverses with "simple" expressions are prefered in most cases, i. e., pseudo-inverses where many entries are zero or one. This can be achieved heuristically, by the following approach:

$\forall \mathbf{A} \in \mathfrak{K}^{m \times n}, \operatorname{rk} \mathbf{A}=m<n \exists \mathbf{V}_{\pi} \in \operatorname{Sym}(n):$ $\mathbf{A V}_{\pi}=(\mathbf{S}, \mathbf{T})$ with $\mathbf{S} \in \mathrm{GL}_{m}(\mathfrak{K})$ such that $\mathbf{A V}_{\pi} \operatorname{row}\left(\mathbf{S}^{-1}, \mathbf{0}\right)=\mathbf{I}_{m}$, i. e., $\mathbf{A}^{+R}=\mathbf{V}_{\pi} \operatorname{row}\left(\mathbf{S}^{-1}, \mathbf{0}\right)$.

Remark 3. The computation of left pseudo-inverses can be deduced from the right counterpart by the following implications:

$\forall \mathbf{A} \in \mathfrak{K}^{m \times n}, \operatorname{rk} \mathbf{A}=n<m: \mathbf{A}^{+\mathrm{L}} \mathbf{A}=\mathbf{I}_{n} \Longrightarrow$ $\mathbf{I}_{n}=\mathbf{A}^{\top}\left(\mathbf{A}^{+\mathrm{L}}\right)^{\top}=\mathbf{A}^{\top}\left(\mathbf{A}^{\top}\right)^{+\mathrm{R}} \Longrightarrow\left(\mathbf{A}^{+\mathrm{L}}\right)^{\top}=\left(\mathbf{A}^{\top}\right)^{+R}$ $\Longrightarrow \mathbf{A}^{+\mathrm{L}}=\left(\left(\mathbf{A}^{\top}\right)^{+\mathbf{R}}\right)^{\top}$.

Remark 4. Given a matrix $\mathbf{A} \in \mathfrak{K}^{m \times n}$ with rk $\mathbf{A}=\min (m, n)$, a general pseudo-inverse can be parameterized by

$$
\mathbf{A}^{+}(\lambda)= \begin{cases}\mathbf{A}_{\mathrm{MP}}^{+}+\mathbf{A}^{\perp} \mathbf{M} & \text { for } m \leq n, \\ \mathbf{A}_{\mathrm{MP}}^{+}+\mathbf{M} \mathbf{A}^{\perp} & \text { for } n<m,\end{cases}
$$

with the Moore-Penrose pseudo-inverse

$$
\mathbf{A}_{\mathrm{MP}}^{+}= \begin{cases}\mathbf{A}^{\top}\left(\mathbf{A} \mathbf{A}^{\top}\right)^{-1} & \text { for } m \leq n \\ \left(\mathbf{A}^{\top} \mathbf{A}\right)^{-1} \mathbf{A}^{\top} & \text { for } n<m\end{cases}
$$

an arbitrary matrix $\mathbf{M} \in \mathfrak{K}^{(\max (m, n)-\mathrm{rk} \mathbf{A}) \times \min (m, n)}$ and an orthogonal complement $\mathbf{A}^{\perp}$ which can be described geometrically by

$$
\begin{array}{crl}
\operatorname{im}\left(\mathbf{A}^{\perp}\right) & =\operatorname{ker} \mathbf{A} & \text { for } m \leq n, \\
\operatorname{im}\left(\left(\mathbf{A}^{\perp}\right)^{\top}\right) & =\operatorname{ker}\left(\mathbf{A}^{\top}\right) & \text { for } n<m .
\end{array}
$$

The columns (rows) of the matrix $\mathbf{A}^{\perp}$ are a basis of $\operatorname{im}\left(\mathbf{A}^{\perp}\right)$ for $m \leq n$ (for $\left.n<m\right)$.

Definition 2. A polynomial matrix $\mathbf{A}(\lambda) \in \mathfrak{K}^{n \times n}[\lambda]$ is called unimodular iff an inverse $\mathbf{A}^{-1}(\lambda) \in \mathfrak{K}^{n \times n}[\lambda]$ exists. We denote by $\mathcal{U}_{n}[\lambda]$ the set of unimodular $n \times n$ matrices.

Definition 3. Let $\mathbf{A}(\lambda) \in \mathfrak{K}^{m \times n}[\lambda]$. The following holds (Cohn, 1985; Lévine, 2011): $\exists \mathbf{L}(\lambda) \in \mathcal{U}_{m}[\lambda], \mathbf{R}(\lambda) \in$ $\mathcal{U}_{n}[\lambda]:$

$$
\mathbf{L}(\lambda) \mathbf{A}(\lambda) \mathbf{R}(\lambda)=\left(\begin{array}{cc}
\Delta(\lambda) & \mathbf{0}_{r \times(n-r)} \\
\mathbf{0}_{(m-r) \times r} & \mathbf{0}_{(m-r) \times(n-r)}
\end{array}\right),
$$

where $\boldsymbol{\Delta}(\lambda) \in \mathfrak{K}^{r \times r}[\lambda]$ with the rank $r \leq \min (m, n)$ denotes a diagonal matrix and the right-hand side of equation (9) is called the Smith normal form of $\mathbf{A}(\lambda)$.

Definition 4. Let $\mathbf{A}(\lambda) \in \mathfrak{K}^{m \times n}[\lambda] . \quad \mathbf{A}(\lambda)$ is called hyper-regular iff its Smith normal form yields $\Delta(\lambda)=$ $\mathbf{I}_{\min (m, n)}$.

Corollary 1. A matrix $\mathbf{A} \in \mathfrak{K}^{n \times n}[\lambda]$ is hyper-regular iff it is unimodular.

Corollary 2. Let $\mathbf{A}(\lambda) \in \mathfrak{K}^{m \times n}[\lambda]$ with $m<n$ be hyper-regular. Then

$$
\exists \mathbf{R}(\lambda) \in \mathcal{U}_{n}[\lambda]: \mathbf{A}(\lambda) \mathbf{R}(\lambda)=\left(\mathbf{I}_{m}, \mathbf{0}\right) .
$$

Proof. See the works of Antritter and Middeke (2011) or Middeke (2011).

Remark 5. In general, the rank of a symbolic matrix depends on the point of evaluation (see Footnote 2). If a submatrix has full rank at one point of evaluation, the set of points where the rank diminishes constitutes a meagre set, i.e., a set of first category in the sense of Baire. This means, that we have maximum rank for almost all points of evaluation. In practice, we can compute the rank of a matrix with symbolic entries by substituting a random number for each symbol and thus transforming the symbolic task into a numerical one, where the challenge is to specify whether or not numerical entries are zero. This problem depends on the 
precision of the floating point variables in use. We have implemented the toolbox in the computer algebra system SymPy (Meurer et al., 2017) which allows evaluation with arbitrary precision and thus enables us to distinguish between numerical noise and small, but finite numbers, such that this problem can be circumvented, too. For more details on the implementation of this, see the works of Knoll (2016, p. 176), or Knoll and Fritzsche (2017).

\section{Hyper-regularity}

In order to check the hyper-regularity of a matrix with entries in $\mathfrak{K}[\lambda]$ we show that left (or right) invertibility is necessary and sufficient. Due to the non-commutativity of $\mathfrak{K}[\lambda]$ we need to distinguish between right and left polynomial matrices, i. e., matrices of the form $\mathbf{A}(\lambda)=$ $\sum_{i=0}^{\alpha} \mathbf{A}_{i} \lambda^{i}$ and $\mathbf{A}(\lambda)=\sum_{i=0}^{\alpha} \lambda^{i} \mathbf{A}_{i}$ (which of course can be transformed into each other). To prove right invertibility, we can use a matrix of an arbitrary (but fixed) order $\beta$ as an ansatz, multiply both matrices and left-shift the differential operator $\lambda$. Since the product of a matrix and its hyper-regular inverse is the unit matrix, comparing coefficients with the unit matrix results in a system of linear equations. Using a left polynomial matrix as an ansatz is necessary to prevent from shifting across the unknown coefficients. We define an operator that simplifies the assembly of the resulting linear equations and thus circumvents non-commutative shifting operations. The number of linear equations depends on the order of the ansatz and is unknown in general. We can prove solvability of these equations by rank conditions, which can be done efficiently using computer algebra systems (see Remark 5). Checking left invertibility follows a similar approach.

Corollary 3. Let $\mathbf{A}(\lambda) \in \mathfrak{K}^{m \times n}[\lambda]$. Then

$$
\begin{aligned}
& \mathbf{A}(\lambda) \text { is hyper-regular } \\
& \Leftrightarrow \begin{cases}\mathbf{A}(\lambda) \text { right invertible } & \text { for } m \leq n, \\
\mathbf{A}(\lambda) \text { left invertible } & \text { for } m \geq n .\end{cases}
\end{aligned}
$$

Proof. Due to the Smith normal form of $\mathbf{A}(\lambda)$, hyper-regularity requires $\boldsymbol{\Delta}(\lambda)=\mathbf{I}$, which is equivalent to the existence of a matrix $\mathbf{M}(\lambda) \in \mathfrak{K}^{n \times m}[\lambda]$ such that $\mathbf{A}(\lambda) \mathbf{M}(\lambda)=\mathbf{I}_{m}$ for $m \leq n$ and $\mathbf{M}(\lambda) \mathbf{A}(\lambda)=\mathbf{I}_{n}$ for $n \leq m$, respectively.

3.1. Conditions for right and left invertibility. The following definitions introduce the operators $\mathcal{H}_{\beta}^{\mathrm{R}}$ and $\mathcal{H}_{\beta}^{L}$ which operate on the coefficient matrices of the polynomial matrix and help in assembling systems of linear equations leading to simple rank conditions to prove hyper-regularity. These operators can easily be implemented.
Definition 5. Let

$$
\mathbf{A}(\lambda)=\sum_{i=0}^{\alpha} \mathbf{A}_{i} \lambda^{i}
$$

with $\mathbf{A}_{i} \in \mathfrak{K}^{m \times n}, m \leq n$ and $\beta \in \mathbb{N}$. The operator ${ }^{3}$

$$
\mathcal{H}_{\beta}^{\mathrm{R}}: \mathfrak{K}^{m \times n}[\lambda] \hookrightarrow \mathfrak{K}^{m(\alpha+\beta+1) \times n(\beta+1)}
$$

is defined by

$$
\left(\mathbf{A}_{0}, \ldots, \mathbf{A}_{\alpha}\right) \mapsto \sum_{i=0}^{\alpha} \mathfrak{H}_{\beta, i}^{\mathrm{R}}\left(\mathbf{A}_{i}\right)
$$

with the matrix

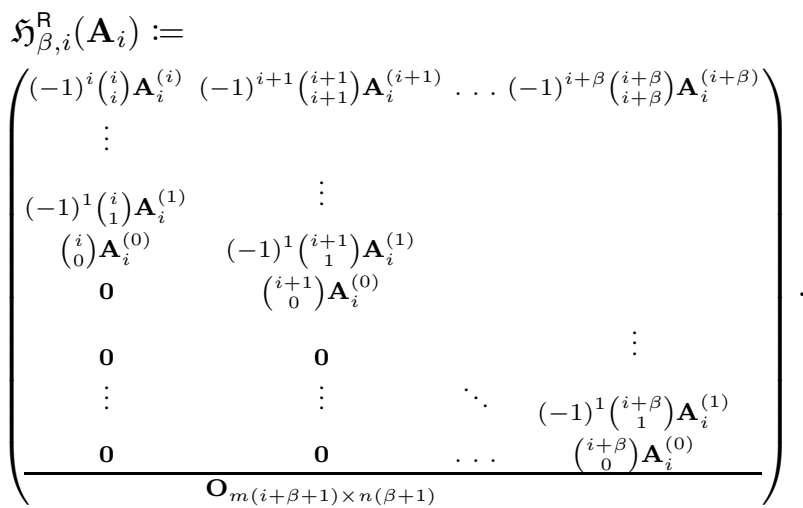

Further, we define

$$
\overline{\mathcal{H}}_{\beta}^{\mathrm{R}} \mathbf{A}(\lambda):=\left(\mathcal{H}_{\beta}^{\mathrm{R}} \mathbf{A}(\lambda),\left(\begin{array}{c}
\mathbf{I}_{m} \\
\mathbf{0}
\end{array}\right)\right) .
$$

Definition 6. Let

$$
\mathbf{A}(\lambda)=\sum_{i=0}^{\alpha} \lambda^{i} \mathbf{A}_{i}
$$

with $\mathbf{A}_{i} \in \mathfrak{K}^{n \times m}, m \leq n$ and $\beta \in \mathbb{N}$. The operator

$$
\mathcal{H}_{\beta}^{\mathrm{L}}: \mathfrak{K}^{n \times m}[\lambda] \hookrightarrow \mathfrak{K}^{n(\beta+1) \times m(\alpha+\beta+1)}
$$

is defined by

$$
\left(\mathbf{A}_{0}, \ldots, \mathbf{A}_{\alpha}\right) \mapsto \sum_{i=0}^{\alpha} \mathfrak{H}_{\beta, i}^{\llcorner}\left(\mathbf{A}_{i}\right)
$$

with the matrix

$\mathfrak{H}_{\beta, i}^{\mathrm{L}}\left(\mathbf{A}_{i}\right):=$

$\left(\begin{array}{cccccc}\left(\begin{array}{c}i \\ 0\end{array}\right) \mathbf{A}_{i}^{(i)} & \ldots & \left(\begin{array}{c}i \\ i\end{array}\right) \mathbf{A}_{i}^{(0)} & & & \\ \left(\begin{array}{c}i+1 \\ 0\end{array}\right) \mathbf{A}_{i}^{(i+1)} & \ldots & \left(\begin{array}{c}i+1 \\ i\end{array}\right) \mathbf{A}_{i}^{(1)} & \left(\begin{array}{c}i+1 \\ i+1\end{array}\right) \mathbf{A}_{i}^{(0)} & & 0 \\ \vdots & & & & \ddots & \\ \left(\begin{array}{c}i+\beta \\ 0\end{array}\right) \mathbf{A}_{i}^{(i+\beta)} & \ldots & \left(\begin{array}{c}i+\beta \\ i\end{array}\right) \mathbf{A}_{i}^{(\beta)} & \left(\begin{array}{c}i+\beta \\ i+1\end{array}\right) \mathbf{A}_{i}^{(\beta-1)} & \ldots & \left(\begin{array}{c}i+\beta \\ i+\beta\end{array}\right) \mathbf{A}_{i}^{(0)}\end{array} \mid \mathbf{O}_{p \times q}\right)$,

where $p=m(\beta+1)$ and $q=n(\alpha-i)$. We define

\footnotetext{
${ }^{3}$ The injective character of the mapping is denoted by $\hookrightarrow$.
} 


$$
\overline{\mathcal{H}}_{\beta}^{\llcorner} \mathbf{A}(\lambda):=\left(\begin{array}{c}
\mathcal{H}_{\beta}^{\llcorner} \mathbf{A}(\lambda) \\
\mathbf{I}_{m}, \mathbf{0}
\end{array}\right) .
$$

Proposition 1. Let

$$
\mathbf{A}(\lambda)=\sum_{i=0}^{\alpha} \mathbf{A}_{i} \lambda^{i}
$$

with $\mathbf{A}_{i} \in \mathfrak{K}^{m \times n}, m \leq n . \mathbf{A}(\lambda)$ is right invertible iff $\exists \beta \in\{0, \ldots, m \alpha\}$ such that

$$
\operatorname{rk}\left(\mathcal{H}_{\beta}^{\mathrm{R}} \mathbf{A}(\lambda)\right)=\operatorname{rk}\left(\overline{\mathcal{H}}_{\beta}^{\mathrm{R}} \mathbf{A}(\lambda)\right) .
$$

Proof. Right invertibility of $\mathbf{A}(\lambda)$ implies the existence of a matrix $\mathbf{B}(\lambda) \in \mathfrak{K}^{n \times m}[\lambda]$ such that $\mathbf{A}(\lambda) \mathbf{B}(\lambda)=\mathbf{I}_{m}$. With $\mathbf{B}(\lambda)=\sum_{j=0}^{\beta} \lambda^{j} \mathbf{B}_{j}, \beta \in \mathbb{N}$ and Eqn. (4) this means

$$
\begin{aligned}
\mathbf{I}_{m}= & \mathbf{A}(\lambda) \mathbf{B}(\lambda) \\
= & \sum_{i=0}^{\alpha} \mathbf{A}_{i} \lambda^{i} \sum_{j=0}^{\beta} \lambda^{j} \mathbf{B}_{j} \\
= & \sum_{i=0}^{\alpha} \sum_{j=0}^{\beta} \mathbf{A}_{i} \lambda^{i} \lambda^{j} \mathbf{B}_{j} \\
= & \sum_{i=0}^{\alpha} \sum_{j=0}^{\beta} \mathbf{A}_{i} \lambda^{i+j} \mathbf{B}_{j} \\
= & \sum_{i=0}^{\alpha} \sum_{j=0}^{\beta} \sum_{k=0}^{i+j}(-1)^{k}\left(\begin{array}{c}
i+j \\
k
\end{array}\right) \lambda^{i+j-k} \mathbf{A}_{i}^{(k)} \mathbf{B}_{j} \\
= & \sum_{j=0}^{\beta} \sum_{i=0}^{\alpha} \sum_{k=0}^{i+j} \lambda^{i+j-k}(-1)^{k}\left(\begin{array}{c}
i+j \\
k
\end{array}\right) \mathbf{A}_{i}^{(k)} \mathbf{B}_{j} \\
= & \sum_{i=0}^{\alpha}\left(\sum_{k=0}^{i}(-1)^{k}\left(\begin{array}{c}
i \\
k
\end{array}\right) \lambda^{i-k} \mathbf{A}_{i}^{(k)}, \ldots,\right. \\
& \left.\sum_{k=0}^{i+\beta}(-1)^{k}\left(\begin{array}{c}
i+\beta \\
k
\end{array}\right) \lambda^{i+\beta-k} \mathbf{A}_{i}^{(k)}\right) \cdot\left(\begin{array}{c}
\mathbf{B}_{0} \\
\vdots \\
\mathbf{B}_{\beta}
\end{array}\right) \\
= & \left(\mathbf{I}_{m}, \lambda \mathbf{I}_{m}, \ldots, \lambda^{\alpha+\beta} \mathbf{I}_{m}\right) \\
& \cdot \sum_{i=0}^{\alpha} \mathfrak{H}_{\beta, i}^{\mathrm{R}}\left(\mathbf{A}_{i}\right) \cdot\left(\begin{array}{c}
\mathbf{B}_{0} \\
\vdots \\
\mathbf{B}_{\beta}
\end{array}\right) .
\end{aligned}
$$

With the operator $\mathcal{H}_{\beta}^{\mathrm{R}}$ this leads to

$$
\mathbf{I}_{m}=\left(\mathbf{I}_{m}, \lambda \mathbf{I}_{m}, \ldots, \lambda^{\alpha+\beta} \mathbf{I}_{m}\right) \cdot \mathcal{H}_{\beta}^{\mathrm{R}} \mathbf{A}(\lambda)\left(\begin{array}{c}
\mathbf{B}_{0} \\
\vdots \\
\mathbf{B}_{\beta}
\end{array}\right),
$$

i. e., to the system of linear equations

$$
\mathcal{H}_{\beta}^{\mathrm{R}} \mathbf{A}(\lambda)\left(\begin{array}{c}
\mathbf{B}_{0} \\
\vdots \\
\mathbf{B}_{\beta}
\end{array}\right)=\left(\begin{array}{c}
\mathbf{I}_{m} \\
\mathbf{0}
\end{array}\right) .
$$

Equation (16) has solutions, iff

$$
\operatorname{rk}\left(\mathcal{H}_{\beta}^{\mathrm{R}} \mathbf{A}(\lambda)\right)=\operatorname{rk}\left(\mathcal{H}_{\beta}^{\mathrm{R}} \mathbf{A}(\lambda),\left(\begin{array}{c}
\mathbf{I}_{m} \\
0
\end{array}\right)\right)
$$

which is equal to condition (13). The degree of $\mathbf{B}(\lambda)$ is bounded with $\beta \leq m \alpha$ (Beckermann et al., 2006), so invertibility can be verified in finitely many steps.

\section{Corollary 4. Let}

$$
\mathbf{A}(\lambda)=\sum_{i=0}^{\alpha} \mathbf{A}_{i} \lambda^{i}
$$

with $\mathbf{A}_{i} \in \mathfrak{K}^{m \times n}$ and

$$
\mathcal{H}_{\beta}^{\mathrm{R}} \mathbf{A}(\lambda)=\sum_{i=0}^{\alpha} \mathfrak{H}_{\beta, i}^{\mathrm{R}}\left(\mathbf{A}_{i}\right)
$$

with

$$
\operatorname{rk}\left(\mathcal{H}_{\beta}^{\mathrm{R}} \mathbf{A}(\lambda)\right)=\operatorname{rk}\left(\overline{\mathcal{H}}_{\beta}^{\mathrm{R}} \mathbf{A}(\lambda)\right)
$$

for $\beta \in \mathbb{N}$. A right inverse

$$
\mathbf{B}(\lambda)=\sum_{i=0}^{\beta} \lambda^{i} \mathbf{B}_{i}
$$

of $\mathbf{A}(\lambda)$ with $\mathbf{B}_{i} \in \mathfrak{K}^{n \times m}$ can be determined by

$$
\mathbf{B}(\lambda)=\left(\mathbf{I}_{n}, \lambda \mathbf{I}_{n}, \ldots, \lambda^{\beta} \mathbf{I}_{n}\right)\left(\mathcal{H}_{\beta}^{\mathrm{R}} \mathbf{A}(\lambda)\right)^{+}\left(\begin{array}{c}
\mathbf{I}_{m} \\
\mathbf{0}
\end{array}\right) .
$$

Proof. A solution to (16) is

$$
\left(\begin{array}{c}
\mathbf{B}_{0} \\
\vdots \\
\mathbf{B}_{\beta}
\end{array}\right)=\left(\mathcal{H}_{\beta}^{\mathrm{R}} \mathbf{A}(\lambda)\right)^{+}\left(\begin{array}{c}
\mathbf{I}_{m} \\
\mathbf{0}
\end{array}\right) \text {. }
$$

Premultiplying the result by $\left(\mathbf{I}_{n}, \lambda \mathbf{I}_{n}, \ldots, \lambda^{\beta} \mathbf{I}_{n}\right)$ completes the proof.

Similar to right invertibility, we can state a condition for left invertibility:

\section{Proposition 2. Let}

$$
\mathbf{A}(\lambda)=\sum_{i=0}^{\alpha} \lambda^{i} \mathbf{A}_{i}
$$

with $\mathbf{A}_{i} \in \mathfrak{K}^{n \times m}, n \geq m$. $\mathbf{A}(\lambda)$ is left invertible iff $\exists \beta \in\{0, \ldots, m \alpha\}$ such that

$$
\operatorname{rk}\left(\mathcal{H}_{\beta}^{\llcorner} \mathbf{A}(\lambda)\right)=\operatorname{rk}\left(\overline{\mathcal{H}}_{\beta}^{\mathrm{L}} \mathbf{A}(\lambda)\right) .
$$


Proof. Left invertibility implies the existence of a matrix $\mathbf{B}(\lambda) \in \mathfrak{K}^{m \times n}[\lambda]$, such that $\mathbf{B}(\lambda) \mathbf{A}(\lambda)=\mathbf{I}_{m}$ holds. With $\mathbf{B}(\lambda)=\sum_{i=0}^{\beta} \mathbf{B}_{i} \lambda^{i}$ for some $\beta \in \mathbb{N}$ and Eqn. (2), we get

$$
\begin{aligned}
& \mathbf{I}_{m}=\mathbf{B}(\lambda) \mathbf{A}(\lambda) \\
& =\sum_{i=0}^{\beta} \mathbf{B}_{i} \lambda^{i} \sum_{j=0}^{\alpha} \lambda^{j} \mathbf{A}_{j} \\
& =\sum_{i=0}^{\beta} \mathbf{B}_{i} \sum_{j=0}^{\alpha} \lambda^{i} \lambda^{j} \mathbf{A}_{j} \\
& =\sum_{i=0}^{\beta} \mathbf{B}_{i} \sum_{j=0}^{\alpha} \lambda^{i+j} \mathbf{A}_{j} \\
& =\sum_{i=0}^{\beta} \mathbf{B}_{i} \sum_{j=0}^{\alpha} \sum_{k=0}^{i+j}\left(\begin{array}{c}
i+j \\
k
\end{array}\right) \mathbf{A}_{j}^{(i+j-k)} \lambda^{k} \\
& =\left(\mathbf{B}_{0}, \ldots, \mathbf{B}_{\beta}\right) \sum_{j=0}^{\alpha}\left(\begin{array}{c}
\sum_{k=0}^{j}\left(\begin{array}{c}
j \\
k
\end{array}\right) \mathbf{A}_{j}^{(j-k)} \lambda^{k} \\
\sum_{k=0}^{1+j}\left(\begin{array}{c}
1+j \\
k
\end{array}\right) \mathbf{A}_{j}^{(1+j-k)} \lambda^{k} \\
\vdots \\
\sum_{k=0}^{\beta+j}\left(\begin{array}{c}
\beta+j \\
k
\end{array}\right) \mathbf{A}_{j}^{(\beta+j-k)} \lambda^{k}
\end{array}\right) \\
& =\left(\mathbf{B}_{0}, \ldots, \mathbf{B}_{\beta}\right) \text {. } \\
& \sum_{j=0}^{\alpha}\left(\begin{array}{c}
\left(\begin{array}{c}
j \\
0
\end{array}\right) \mathbf{A}_{j}^{(j)}+\left(\begin{array}{c}
j \\
1
\end{array}\right) \mathbf{A}_{j}^{(j-1)} \lambda+\cdots+\left(\begin{array}{c}
j \\
j
\end{array}\right) \mathbf{A}_{j} \lambda^{j} \\
\left(\begin{array}{c}
1+j \\
0
\end{array}\right) \mathbf{A}_{j}^{(1+j)}+\left(\begin{array}{c}
1+j \\
1
\end{array}\right) \mathbf{A}_{j}^{(j)} \lambda+\cdots+\left(\begin{array}{c}
1+j \\
1+j
\end{array}\right) \mathbf{A}_{j} \lambda^{1+j} \\
\vdots \\
\left(\begin{array}{c}
\beta+j \\
0
\end{array}\right) \mathbf{A}_{j}^{(\beta+j)}+\left(\begin{array}{c}
\beta+j \\
1
\end{array}\right) \mathbf{A}_{j}^{(\beta+j-1)} \lambda+\cdots+\left(\begin{array}{c}
\beta+j \\
\beta+j
\end{array}\right) \mathbf{A}_{j} \lambda^{\beta+j}
\end{array}\right) \\
& =\left(\mathbf{B}_{0}, \ldots, \mathbf{B}_{\beta}\right) \sum_{j=0}^{\alpha}\left(\begin{array}{cc}
\left(\begin{array}{c}
j \\
0
\end{array}\right) \mathbf{A}_{j}^{(i)} & \ldots \\
\left(\begin{array}{c}
1+j \\
0
\end{array}\right) \mathbf{A}_{j}^{(1+j)} & \ldots \\
\vdots & \\
\left(\begin{array}{c}
\beta+j \\
0
\end{array}\right) \mathbf{A}_{j}^{(\beta+j)} & \ldots
\end{array}\right. \\
& \left(\begin{array}{l}
j \\
j
\end{array}\right) \mathbf{A}_{j}^{(0)} \\
& \left(\begin{array}{c}
1+j \\
j
\end{array}\right) \mathbf{A}_{j}^{(1)} \quad\left(\begin{array}{c}
1+j \\
1+j
\end{array}\right) \mathbf{A}_{j}^{(0)} \\
& \left.\left(\begin{array}{c}
\beta+j \\
j
\end{array}\right) \mathbf{A}_{j}^{(\beta)} \quad\left(\begin{array}{c}
\beta+j \\
1+j
\end{array}\right) \mathbf{A}_{j}^{(\beta-1)} \quad \ldots \quad\left(\begin{array}{c}
\beta+j \\
1+j
\end{array}\right) \mathbf{A}_{j}^{0}\right) \\
& \left(\begin{array}{c}
\mathbf{I}_{m} \\
\lambda \mathbf{I}_{m} \\
\vdots \\
\lambda^{\beta+j} \mathbf{I}_{m}
\end{array}\right)
\end{aligned}
$$

which, using the operator $\mathcal{H}_{\beta}^{\mathrm{L}}$, leads to

$$
\left(\mathbf{B}_{0}, \ldots, \mathbf{B}_{\beta}\right) \mathcal{H}_{\beta}^{\llcorner} \mathbf{A}(\lambda)\left(\begin{array}{c}
\mathbf{I}_{m} \\
\lambda \mathbf{I}_{m} \\
\vdots \\
\lambda^{\alpha+\beta} \mathbf{I}_{m}
\end{array}\right)=\mathbf{I}_{m}
$$

and thus to the system of linear equations

$$
\left(\mathbf{B}_{0}, \ldots, \mathbf{B}_{\beta}\right) \mathcal{H}_{\beta}^{\llcorner} \mathbf{A}(\lambda)=\left(\mathbf{I}_{m}, \mathbf{0}\right)
$$

This equation has solutions iff

$$
\begin{aligned}
\operatorname{rk}\left(\mathcal{H}_{\beta}^{\mathrm{L}} \mathbf{A}(\lambda)\right) & =\operatorname{rk}\left(\begin{array}{c}
\mathcal{H}_{\beta}^{\mathrm{L}} \mathbf{A}(\lambda) \\
\mathbf{I}_{m}, \mathbf{0}
\end{array}\right) \\
& =\operatorname{rk}\left(\overline{\mathcal{H}}_{\beta}^{\mathrm{L}} \mathbf{A}(\lambda)\right)
\end{aligned}
$$

for $\beta \leq m \alpha$ (Beckermann et al., 2006) which completes the proof.

Corollary 5. Let

$$
\mathbf{A}(\lambda)=\sum_{i=0}^{\alpha} \lambda^{i} \mathbf{A}_{i}
$$

with $\mathbf{A}_{i} \in \mathfrak{K}^{n \times m}$ and

$$
\mathcal{H}_{\beta}^{\mathrm{L}} \mathbf{A}(\lambda)=\sum_{i=0}^{\alpha} \mathfrak{H}_{\beta, i}^{\mathrm{L}}\left(\mathbf{A}_{i}\right)
$$

with

$$
\operatorname{rk}\left(\mathcal{H}_{\beta}^{\mathrm{L}} \mathbf{A}(\lambda)\right)=\operatorname{rk}\left(\overline{\mathcal{H}}_{\beta}^{\mathrm{L}} \mathbf{A}(\lambda)\right)
$$

A left inverse

$$
\mathbf{B}(\lambda)=\sum_{i=0}^{\beta} \mathbf{B}_{i} \lambda^{i} \in \mathfrak{K}^{m \times n}[\lambda]
$$

of $\mathbf{A}(\lambda)$ can be determined by

$$
\mathbf{B}(\lambda)=\left(\mathbf{I}_{m}, \mathbf{0}\right)\left(\mathcal{H}_{\beta}^{\mathrm{L}} \mathbf{A}(\lambda)\right)^{+}\left(\begin{array}{c}
\mathbf{I}_{n} \\
\lambda \mathbf{I}_{n} \\
\vdots \\
\lambda^{\alpha+\beta} \mathbf{I}_{n}
\end{array}\right) .
$$

Proof. Postmultiplying Eqn. (23) by $\left(\mathcal{H}_{\beta}^{\mathrm{L}} \mathbf{A}(\lambda)\right)^{+}$results in (25).

\subsection{Conversion of left and right polynomial ma-} trices. Since the computation of hyper-regular inverses of left polynomial matrices as described above yields hyper-regular right polynomial matrices and vice versa, it may be convenient to convert between these.

Let $\mathbf{A}(\lambda)=\sum_{i=0}^{\alpha} \lambda^{i} \mathbf{A}_{i}$ with $\mathbf{A}_{i} \in \mathfrak{K}^{m \times n}$ and $\alpha \in \mathbb{N}$ be a left polynomial matrix, i. e., the differential operator $\lambda$ is premultiplied by the coefficient matrices $\mathbf{A}_{i}$. Using (2), we can derive a formula for converting $\mathbf{A}(\lambda)$ 
into a right polynomial matrix:

$$
\begin{aligned}
& \mathbf{A}(\lambda)=\sum_{i=0}^{\alpha} \lambda^{i} \mathbf{A}_{i} \\
& =\sum_{i=0}^{\alpha} \sum_{j=0}^{i}\left(\begin{array}{l}
i \\
j
\end{array}\right) \mathbf{A}_{i}^{(i-j)} \lambda^{j} \\
& =\left(\mathbf{A}_{0}\right)+\left(\mathbf{A}_{1}^{(1)}+\mathbf{A}_{1} \lambda\right) \\
& +\left(\mathbf{A}_{2}^{(2)}+2 \mathbf{A}_{2}^{(1)} \lambda+\mathbf{A}_{2} \lambda^{2}\right) \\
& +\left(\mathbf{A}_{3}^{(3)}+3 \mathbf{A}_{3}^{(2)} \lambda+3 \mathbf{A}_{3}^{(1)} \lambda^{2}+\mathbf{A}_{3} \lambda^{3}\right)+\ldots \\
& =\left(\mathbf{A}_{0}+\mathbf{A}_{1}^{(1)}+\mathbf{A}_{2}^{(2)}+\ldots\right) \\
& +\left(\mathbf{A}_{1}+2 \mathbf{A}_{2}^{(1)}+3 \mathbf{A}_{3}^{(2)}+4 \mathbf{A}_{4}^{(3)}+\ldots\right) \lambda \\
& +\left(\mathbf{A}_{2}+3 \mathbf{A}_{3}^{(1)}+6 \mathbf{A}_{4}^{(2)}+10 \mathbf{A}_{5}^{(3)}+\ldots\right) \lambda^{2} \\
& +\ldots \\
& =\sum_{i=0}^{\alpha} \mathbf{A}_{i}^{(i)}+\left(\sum_{i=1}^{\alpha}\left(\begin{array}{l}
i \\
1
\end{array}\right) \mathbf{A}_{i}^{(i-1)}\right) \lambda \\
& +\left(\sum_{i=2}^{\alpha}\left(\begin{array}{l}
i \\
2
\end{array}\right) \mathbf{A}_{i}^{(i-2)}\right) \lambda^{2} \\
& +\left(\sum_{i=3}^{\alpha}\left(\begin{array}{l}
i \\
3
\end{array}\right) \mathbf{A}_{i}^{(i-3)}\right) \lambda^{3}+\ldots \\
& =\left(\sum_{i=0}^{\alpha} \mathbf{A}_{i}^{(i)}, \sum_{i=1}^{\alpha}\left(\begin{array}{l}
i \\
1
\end{array}\right) \mathbf{A}_{i}^{(i-1)}, \ldots,\right. \\
& \left.\sum_{i=\alpha}^{\alpha}\left(\begin{array}{c}
i \\
\alpha
\end{array}\right) \mathbf{A}_{i}^{(i-\alpha)}\right)\left(\begin{array}{c}
\mathbf{I}_{n} \\
\lambda \mathbf{I}_{n} \\
\vdots \\
\lambda^{\alpha} \mathbf{I}_{n}
\end{array}\right) .
\end{aligned}
$$

In the same manner, we can derive a formula for converting right polynomial matrices into left polynomial matrices:

Let $\mathbf{A}(\lambda)=\sum_{i=0}^{\alpha} \mathbf{A}_{i} \lambda^{i}$ with $\mathbf{A}_{i} \in \mathfrak{K}^{m \times n}$ and $\alpha \in$ $\mathbb{N}$ be a right polynomial matrix. Using (4), we get

$$
\begin{aligned}
\mathbf{A}(\lambda)= & \sum_{i=0}^{\alpha} \mathbf{A}_{i} \lambda^{i} \\
= & \sum_{i=0}^{\alpha} \sum_{j=0}^{i}(-1)^{j}\left(\begin{array}{c}
i \\
j
\end{array}\right) \lambda^{i-j} \mathbf{A}_{i}^{(j)} \\
= & \left(\mathbf{A}_{0}\right)+\left(\lambda \mathbf{A}_{1}-\mathbf{A}_{1}^{(1)}\right) \\
& +\left(\lambda^{2} \mathbf{A}_{2}-2 \lambda \mathbf{A}_{2}^{(1)}+\mathbf{A}_{2}^{(2)}\right) \\
& +\left(\lambda^{3} \mathbf{A}_{3}-3 \lambda^{2} \mathbf{A}_{3}^{(1)}+3 \lambda \mathbf{A}_{3}^{(2)}-\mathbf{A}_{3}^{(3)}\right)+\ldots \\
= & \sum_{i=0}^{\alpha}(-1)^{i} \mathbf{A}_{i}^{(i)}
\end{aligned}
$$

$$
\begin{aligned}
& +\lambda\left(\sum_{i=1}^{\alpha}(-1)^{i+1}\left(\begin{array}{l}
i \\
1
\end{array}\right) \mathbf{A}_{i}^{(i-1)}\right) \\
& +\lambda^{2}\left(\sum_{i=2}^{\alpha}(-1)^{i+2}\left(\begin{array}{l}
i \\
2
\end{array}\right) \mathbf{A}_{i}^{(i-2)}\right)+\ldots \\
= & \left(\mathbf{I}_{m}, \lambda \mathbf{I}_{m}, \ldots, \lambda^{\alpha} \mathbf{I}_{m}\right) \\
& \cdot\left(\begin{array}{c}
\sum_{i=0}^{\alpha}(-1)^{i}\left(\begin{array}{c}
i \\
0
\end{array}\right) \mathbf{A}_{i}^{(i)} \\
\sum_{i=1}^{\alpha}(-1)^{i+1}\left(\begin{array}{c}
i \\
1
\end{array}\right) \mathbf{A}_{i}^{(i-1)} \\
\vdots \\
\sum_{i=\alpha}^{\alpha}(-1)^{i+\alpha}\left(\begin{array}{c}
i \\
\alpha
\end{array}\right) \mathbf{A}_{i}^{(i-\alpha)}
\end{array}\right)
\end{aligned}
$$

Example 1. (Hyper-regular right inverse) Let

$$
\begin{aligned}
\mathbf{A}(\lambda) & =\left(\begin{array}{ll}
1+\lambda+\lambda^{2} & \dot{x}_{1}+x_{1} \lambda
\end{array}\right) \\
& =\underbrace{\left(\begin{array}{ll}
1 & \dot{x}_{1}
\end{array}\right)}_{=: \mathbf{A}_{0}}+\underbrace{\left(\begin{array}{ll}
1 & x_{1}
\end{array}\right)}_{=: \mathbf{A}_{1}} \lambda+\underbrace{\left(\begin{array}{ll}
1 & 0
\end{array}\right)}_{=: \mathbf{A}_{2}} \lambda^{2} .
\end{aligned}
$$

$\mathbf{A}(\lambda)$ is a right polynomial matrix already, so we can check the condition (13) which yields $\operatorname{rk}\left(\mathcal{H}_{\beta}^{\mathrm{R}} \mathbf{A}(\lambda)\right)=$ $\operatorname{rk}\left(\overline{\mathcal{H}}_{\beta}^{\mathrm{R}} \mathbf{A}(\lambda)\right)$ for $\beta=1$. We get

$$
\begin{aligned}
& \mathcal{H}_{1}^{\mathrm{R}} \mathbf{A}(\lambda)=\mathfrak{H}_{1,0}^{\mathrm{R}}\left(\mathbf{A}_{0}\right)+\mathfrak{H}_{1,1}^{\mathrm{R}}\left(\mathbf{A}_{1}\right)+\mathfrak{H}_{1,2}^{\mathrm{R}}\left(\mathbf{A}_{2}\right) \\
& =\left(\begin{array}{cc}
\mathbf{A}_{0} & -\dot{\mathbf{A}}_{0} \\
\mathbf{0} & \mathbf{A}_{0} \\
\mathbf{0} & \mathbf{0} \\
\mathbf{0} & \mathbf{0}
\end{array}\right) \\
& +\left(\begin{array}{cc}
-\dot{\mathbf{A}}_{1} & \ddot{\mathbf{A}}_{1} \\
\mathbf{A}_{1} & -2 \dot{\mathbf{A}}_{1} \\
\mathbf{0} & \mathbf{A}_{1} \\
\mathbf{0} & \mathbf{0}
\end{array}\right)+\left(\begin{array}{cc}
\ddot{\mathbf{A}}_{2} & -\dddot{\mathbf{A}}_{2} \\
-2 \dot{\mathbf{A}}_{2} & 3 \ddot{\mathbf{A}}_{2} \\
\mathbf{A}_{2} & -3 \dot{\mathbf{A}}_{2} \\
\mathbf{0} & \mathbf{A}_{2}
\end{array}\right) \\
& =\left(\begin{array}{cc|cc}
1 & \dot{x}_{1} & 0 & -\ddot{x}_{1} \\
\hline 0 & 0 & 1 & \dot{x}_{1} \\
\hline 0 & 0 & 0 & 0 \\
\hline 0 & 0 & 0 & 0
\end{array}\right) \\
& +\left(\begin{array}{cc|cc}
0 & -\dot{x}_{1} & 0 & \ddot{x}_{1} \\
\hline 1 & x_{1} & 0 & -2 \dot{x}_{1} \\
\hline 0 & 0 & 1 & x_{1} \\
\hline 0 & 0 & 0 & 0
\end{array}\right) \\
& +\left(\begin{array}{ll|ll}
0 & 0 & 0 & 0 \\
\hline 0 & 0 & 0 & 0 \\
\hline 1 & 0 & 0 & 0 \\
\hline 0 & 0 & 1 & 0
\end{array}\right) \\
& =\left(\begin{array}{cc|cc}
1 & 0 & 0 & 0 \\
\hline 1 & x_{1} & 1 & -\dot{x}_{1} \\
\hline 1 & 0 & 1 & x_{1} \\
\hline 0 & 0 & 1 & 0
\end{array}\right)
\end{aligned}
$$

with $\operatorname{rk}\left(\mathcal{H}_{1}^{\mathrm{R}} \mathbf{A}(\lambda)\right)=4$ such that we can calculate a right 
inverse

$$
\begin{aligned}
\left(\begin{array}{l}
\mathbf{B}_{0} \\
\mathbf{B}_{1}
\end{array}\right) & =\left(\mathcal{H}_{1}^{\mathrm{R}} \mathbf{A}(\lambda)\right)^{-1}\left(\begin{array}{c}
\mathbf{I}_{m} \\
\mathbf{0}
\end{array}\right) \\
& =\left(\begin{array}{cccc}
1 & 0 & 0 & 0 \\
-\frac{x_{1}+\dot{x}_{1}}{x_{1}^{2}} & \frac{1}{x_{1}} & \frac{\dot{x}_{1}}{x_{1}^{2}} & -\frac{x_{1}+\dot{x}_{1}}{x_{1}^{2}} \\
0 & 0 & 0 & 1 \\
-\frac{1}{x_{1}} & 0 & \frac{1}{x_{1}} & -\frac{1}{x_{1}}
\end{array}\right)\left(\begin{array}{l}
1 \\
0 \\
0 \\
0
\end{array}\right) \\
& =\left(\begin{array}{c}
1 \\
-\frac{x_{1}+\dot{x}_{1}}{x_{1}^{2}} \\
0 \\
-\frac{1}{x_{1}}
\end{array}\right),
\end{aligned}
$$

i.e.,

$$
\mathbf{B}(\lambda)=\mathbf{B}_{0}+\lambda \mathbf{B}_{1}=\left(\begin{array}{c}
1 \\
-\frac{x_{1}+\dot{x}_{1}}{x_{1}^{2}}-\lambda \frac{1}{x_{1}}
\end{array}\right) .
$$

Example 2. (Hyper-regular left inverse) Let

$$
\mathbf{A}(\lambda)=\left(\begin{array}{c}
1+\lambda+\lambda^{2} \\
x_{2}+x_{2} \lambda
\end{array}\right) \in \mathfrak{K}^{2 \times 1}[\lambda] .
$$

Converting $\mathbf{A}(\lambda)$ into a left polynomial matrix by applying equation (27) yields

$$
\begin{aligned}
\mathbf{A}(\lambda) & =\left(\begin{array}{c}
1+\lambda+\lambda^{2} \\
x_{2}-\dot{x}_{2}+\lambda x_{2}
\end{array}\right) \\
& =\underbrace{\left(\begin{array}{c}
1 \\
x_{2}-\dot{x}_{2}
\end{array}\right)}_{=: \mathbf{A}_{0}}+\lambda \underbrace{\left(\begin{array}{c}
1 \\
x_{2}
\end{array}\right)}_{=: \mathbf{A}_{1}}+\lambda^{2} \underbrace{\left(\begin{array}{c}
1 \\
0
\end{array}\right)}_{=: \mathbf{A}_{2}} .
\end{aligned}
$$

We can verify that the condition (20) is satisfied for $\beta=1$ and we get

$$
\begin{aligned}
\mathcal{H}_{1}^{\mathrm{L}} \mathbf{A}(\lambda)= & \mathfrak{H}_{1,0}^{\mathrm{L}}\left(\mathbf{A}_{0}\right)+\mathfrak{H}_{1,1}^{\mathrm{L}}\left(\mathbf{A}_{1}\right)+\mathfrak{H}_{1,2}^{\mathrm{L}}\left(\mathbf{A}_{2}\right) \\
= & \left(\begin{array}{cccc}
\mathbf{A}_{0} & \mathbf{0} & \mathbf{0} & \mathbf{0} \\
\dot{\mathbf{A}}_{0} & \mathbf{A}_{0} & \mathbf{0} & \mathbf{0}
\end{array}\right) \\
& +\left(\begin{array}{cccc|c}
\dot{\mathbf{A}}_{1} & \mathbf{A}_{1} & \mathbf{0} & \mathbf{0} \\
\ddot{\mathbf{A}}_{1} & 2 \dot{\mathbf{A}}_{1} & \mathbf{A}_{1} & \mathbf{0}
\end{array}\right) \\
& +\left(\begin{array}{ccc|c|c}
\ddot{\mathbf{A}}_{2} & \dot{\mathbf{A}}_{2} & \mathbf{A}_{2} & \mathbf{0} \\
\dddot{\mathbf{A}}_{2} & 3 \ddot{\mathbf{A}}_{2} & 3 \dot{\mathbf{A}}_{2} & \mathbf{A}_{2}
\end{array}\right) \\
= & \left(\begin{array}{c|c|c|c|c}
1 & & 0 & 0 & 0 \\
x_{2}-\dot{x}_{2} & & 0 & 0 & 0 \\
\hline 0 & & 1 & 0 & 0 \\
\dot{x}_{2}-\ddot{x}_{2} & x_{2}-\dot{x}_{2} & 0 & 0
\end{array}\right) \\
& +\left(\begin{array}{c|c|c|c|c}
0 & 1 & 0 & 0 \\
\dot{x}_{2} & x_{2} & 0 & 0 \\
\hline 0 & 0 & 1 & 0 \\
\ddot{x}_{2} & 2 \dot{x}_{2} & x_{2} & 0
\end{array}\right)
\end{aligned}
$$

$$
\begin{aligned}
& +\left(\begin{array}{c|c|c|c}
0 & 0 & 1 & 0 \\
0 & 0 & 0 & 0 \\
\hline 0 & 0 & 0 & 1 \\
0 & 0 & 0 & 0
\end{array}\right) \\
& =\left(\begin{array}{c|c|c|c}
1 & 1 & 1 & 0 \\
x_{2} & x_{2} & 0 & 0 \\
\hline 0 & 1 & 1 & 1 \\
\dot{x}_{2} & x_{2}+\dot{x}_{2} & x_{2} & 0
\end{array}\right) .
\end{aligned}
$$

Since $\operatorname{rk}\left(\mathcal{H}_{1}^{\llcorner} \mathbf{A}(\lambda)\right)=4$, we get

$$
\begin{aligned}
\left(\mathbf{B}_{0}, \mathbf{B}_{1}\right) & =(1,0,0,0)\left(\mathcal{H}_{1}^{\mathrm{L}} \mathbf{A}(\lambda)\right)^{-1} \\
& =(1,0,0,0)\left(\begin{array}{cccc}
1 & \frac{\dot{x}_{2}}{x_{2}^{2}} & 0 & -\frac{1}{x_{2}} \\
-1 & \frac{1}{x_{2}}-\frac{\dot{x}_{2}}{x_{2}^{2}} & 0 & \frac{1}{x_{2}} \\
1 & -\frac{1}{x_{2}} & 0 & 0 \\
0 & \frac{\dot{x}_{2}}{x_{2}^{2}} & 1 & -\frac{1}{x_{2}}
\end{array}\right) \\
& =\left(\begin{array}{llll}
1 & \frac{\dot{x}_{2}}{x_{2}^{2}} & 0 & -\frac{1}{x_{2}}
\end{array}\right)
\end{aligned}
$$

i. e.,

$$
\mathbf{B}(\lambda)=\mathbf{B}_{0}+\mathbf{B}_{1} \lambda=\left(\begin{array}{ll}
1 & \frac{\dot{x}_{2}}{x_{2}^{2}}-\frac{1}{x_{2}} \lambda
\end{array}\right) .
$$

We can verify the result by checking $\mathbf{B}(\lambda) \mathbf{A}(\lambda)=1$.

\section{Unimodularity}

4.1. Rank conditions to prove unimodularity. While unimodularity implies left and right invertibility, we introduce an operator which allows direct computation of unimodular right polynomial inverses for unimodular right polynomial matrices, i.e., there is no need for left or right conversion as described in Section 3.2 .

In much the same way as in Section 3, we define an operator $\mathcal{T}_{\beta}$ that allows us to state a simple condition for checking unimodularity.

Definition 7. Let

$$
\mathbf{A}(\lambda)=\sum_{i=0}^{\alpha} \mathbf{A}_{i} \lambda^{i}
$$

with $\mathbf{A}_{i} \in \mathfrak{K}^{m \times n}$ and $\beta \in \mathbb{N}$. The operator

$$
\mathcal{T}_{\beta}: \mathfrak{K}^{m \times n}[\lambda] \hookrightarrow \mathfrak{K}^{m(\beta+1) \times n(\alpha+\beta+1)}
$$

is defined by

$$
\left(\mathbf{A}_{0}, \ldots, \mathbf{A}_{\alpha}\right) \mapsto \sum_{i=0}^{\alpha} \mathfrak{T}_{\beta, i}\left(\mathbf{A}_{i}\right)
$$

with the matrix

$$
\begin{aligned}
& \mathfrak{T}_{\beta, i}\left(\mathbf{A}_{i}\right):=
\end{aligned}
$$

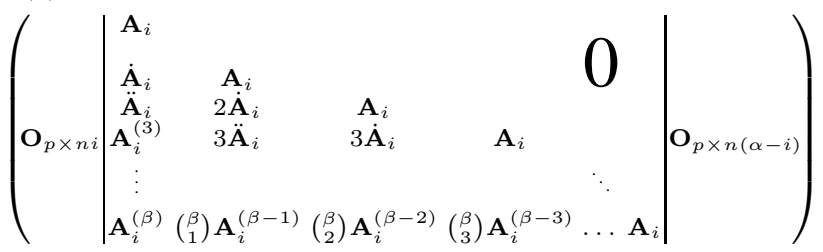


where $p=m(\beta+1)$. Furthermore, we define

$$
\overline{\mathfrak{T}}_{\beta} \mathbf{A}(\lambda):=\left(\begin{array}{c}
\mathcal{T}_{\beta} \mathbf{A}(\lambda) \\
\mathbf{I}_{n}, \mathbf{0}
\end{array}\right) .
$$

The degree bound for unimodular inverses is well known (Lévine, 2011; Ritt, 1935; Ollivier, 1990; Kondratieva et al., 1982; Ollivier and Brahim, 2007) such that we can state the following.

\section{Proposition 3. Let}

$$
\mathbf{A}(\lambda)=\sum_{i=0}^{\alpha} \mathbf{A}_{i} \lambda^{i} \in \mathfrak{K}^{n \times n}[\lambda] .
$$

Then $\mathbf{A}(\lambda) \in \mathcal{U}_{n}[\lambda]$, iff $\exists \beta \in\{0, \ldots, \alpha(n-1)\}$ such that

$$
\operatorname{rk}\left(\mathcal{T}_{\beta} \mathbf{A}(\lambda)\right)=\operatorname{rk}\left(\overline{\mathfrak{T}}_{\beta} \mathbf{A}(\lambda)\right)=n(\beta+1) .
$$

Proof. Let

$$
\mathbf{A}(\lambda)=\sum_{i=0}^{\alpha} \mathbf{A}_{i} \lambda^{i}
$$

with $\mathbf{A}_{i} \in \mathfrak{K}^{n \times n}$. Unimodularity of $\mathbf{A}(\lambda)$ implies the existence of a matrix

$$
\mathbf{B}(\lambda)=\sum_{i=0}^{\beta} \mathbf{B}_{i} \lambda^{i}
$$

with

$$
\mathbf{B}_{i} \in \mathfrak{K}^{n \times n}
$$

such that

$$
\mathbf{B}(\lambda) \mathbf{A}(\lambda)=\mathbf{A}(\lambda) \mathbf{B}(\lambda)=\mathbf{I}_{n} .
$$

Using (2), we get

$$
\begin{aligned}
\mathbf{I}_{n}= & \mathbf{B}(\lambda) \mathbf{A}(\lambda)=\sum_{i=0}^{\beta} \mathbf{B}_{i} \lambda^{i} \sum_{j=0}^{\alpha} \mathbf{A}_{j} \lambda^{j} \\
= & \sum_{i=0}^{\beta} \mathbf{B}_{i} \sum_{j=0}^{\alpha} \lambda^{i} \mathbf{A}_{j} \lambda^{j} \\
= & \sum_{i=0}^{\beta} \mathbf{B}_{i} \sum_{j=0}^{\alpha} \sum_{k=0}^{i}\left(\begin{array}{l}
i \\
k
\end{array}\right) \mathbf{A}_{j}^{(i-k)} \lambda^{k} \lambda^{j} \\
= & \sum_{i=0}^{\beta} \mathbf{B}_{i} \sum_{j=0}^{\alpha} \sum_{k=0}^{i}\left(\begin{array}{l}
i \\
k
\end{array}\right) \mathbf{A}_{j}^{(i-k)} \lambda^{k+j} \\
= & \left(\mathbf{B}_{0}, \ldots, \mathbf{B}_{\beta}\right) \\
& \cdot \sum_{j=0}^{\alpha}\left(\begin{array}{l}
\sum_{k=0}^{0}\left(\begin{array}{l}
0 \\
k
\end{array}\right) \mathbf{A}_{j}^{(0-k)} \lambda^{k+j} \\
\sum_{k=0}^{1}\left(\begin{array}{l}
1 \\
k
\end{array}\right) \mathbf{A}_{j}^{(1-k)} \lambda^{k+j} \\
\vdots \\
\sum_{k=0}^{\beta}\left(\begin{array}{l}
\beta \\
k
\end{array}\right) \mathbf{A}_{j}^{(\beta-k)} \lambda^{k+j}
\end{array}\right)
\end{aligned}
$$

and with the operator $\mathcal{T}_{\beta}$ this is equal to

$$
\mathbf{I}_{n}=\left(\mathbf{B}_{0}, \ldots, \mathbf{B}_{\beta}\right) \mathcal{T}_{\beta} \mathbf{A}(\lambda)\left(\begin{array}{c}
\mathbf{I}_{n} \\
\lambda \mathbf{I}_{n} \\
\vdots \\
\lambda^{\beta} \mathbf{I}_{n}
\end{array}\right)
$$

i.e.,

$$
\left(\mathbf{B}_{0}, \ldots, \mathbf{B}_{\beta}\right) \mathcal{T}_{\beta} \mathbf{A}(\lambda)=\left(\mathbf{I}_{n}, \mathbf{0}\right)
$$

where $\beta \in\{0, \ldots, \alpha(n-1)\}$ (see, e.g., the works of Lévine (2011), Ritt (1935), Ollivier (1990), Kondratieva et al. (1982) or Ollivier and Brahim (2007) for the upper bound of $\beta$ ). This equation has solutions iff

$$
\operatorname{rk}\left(\mathcal{T}_{\beta} \mathbf{A}(\lambda)\right)=\operatorname{rk}\left(\begin{array}{c}
\mathcal{T}_{\beta} \mathbf{A}(\lambda) \\
\mathbf{I}_{n}, \mathbf{0}
\end{array}\right)=\operatorname{rk}\left(\overline{\mathcal{T}}_{\beta} \mathbf{A}(\lambda)\right) .
$$

By requiring

$$
\operatorname{rk}\left(\mathcal{T}_{\beta} \mathbf{A}(\lambda)\right)=n(\beta+1),
$$

we can ensure the existence of exactly one solution and, thus, Eqns. (42) and (43) must hold for $\mathbf{A}(\lambda) \in \mathcal{U}_{n}[\lambda]$.

\section{Corollary 6. Let}

$$
\mathbf{A}(\lambda)=\sum_{i=0}^{\alpha} \mathbf{A}_{i} \lambda^{i} \in \mathcal{U}_{n}[\lambda]
$$

and

$$
\mathcal{T}_{\beta} \mathbf{A}(\lambda)=\sum_{i=0}^{\alpha} \mathfrak{T}_{\beta}\left(\mathbf{A}_{i}\right)
$$

with

$$
\operatorname{rk}\left(\mathcal{T}_{\beta} \mathbf{A}(\lambda)\right)=\operatorname{rk}\left(\overline{\mathfrak{T}}_{\beta} \mathbf{A}(\lambda)\right)=n(\beta+1) .
$$

The coefficient matrices of the inverse

$$
\mathbf{B}(\lambda)=\sum_{i=0}^{\beta} \mathbf{B}_{i} \lambda^{i} \in \mathcal{U}_{n}[\lambda]
$$

of $\mathbf{A}(\lambda)$ can be determined by

$$
\left(\mathbf{B}_{0}, \ldots, \mathbf{B}_{\beta}\right)=\left(\mathbf{I}_{n}, \mathbf{0}\right)\left(\mathcal{T}_{\beta} \mathbf{A}(\lambda)\right)^{+\mathrm{R}}
$$

Proof. Postmultiplying (41) by $\left(\mathcal{T}_{\beta} \mathbf{A}(\lambda)\right)^{+\mathrm{R}}$ leads to (44).

Remark 6. If (13), (20) or (38) is satisfied for some $\beta \in \mathbb{N}$, then it is satisfied for any $\tilde{\beta} \in \mathbb{N}_{\geq \beta}$, too.

Remark 7. Similar conditions for unimodularity could be formulated using the operator $\mathcal{H}_{\beta}^{L}$ and $\mathcal{H}_{\beta}^{\mathrm{R}}$ instead of $\mathcal{T}_{\beta}$, since unimodularity implies right and left invertibility. 
Example 3. $(\alpha=1)$ Let

$$
\begin{aligned}
\mathbf{A}(\lambda) & =\left(\begin{array}{ccc}
-\dot{x}_{2} \lambda & -\dot{x}_{1} \lambda & \lambda \\
\dot{x}_{2} & 0 & -1 \\
0 & 1 & 0
\end{array}\right) \\
& =\underbrace{\left(\begin{array}{ccc}
0 & 0 & 0 \\
\dot{x}_{2} & 0 & -1 \\
0 & 1 & 0
\end{array}\right)}_{=: \mathbf{A}_{0}}+\underbrace{\left(\begin{array}{ccc}
-\dot{x}_{2} & -\dot{x}_{1} & 1 \\
0 & 0 & 0 \\
0 & 0 & 0
\end{array}\right)}_{=: \mathbf{A}_{1}} \lambda .
\end{aligned}
$$

For $\beta=0$ we get

$$
\mathfrak{T}_{0,0}\left(\mathbf{A}_{0}\right)=\left(\begin{array}{ll}
\mathbf{A}_{0} & \mathbf{0}
\end{array}\right), \quad \mathfrak{T}_{0,1}\left(\mathbf{A}_{1}\right)=\left(\begin{array}{ll}
\mathbf{0} & \mathbf{A}_{1}
\end{array}\right),
$$

which results in

$$
\begin{aligned}
\mathcal{T}_{0} \mathbf{A}(\lambda) & =\sum_{i=0}^{1} \mathfrak{T}_{0, i}\left(\mathbf{A}_{i}\right) \\
& =\left(\begin{array}{ccc|ccc}
\mathbf{A}_{0} & \mathbf{A}_{1}
\end{array}\right) \\
& =\left(\begin{array}{ccc|ccc}
0 & 0 & 0 & -\dot{x}_{2} & -\dot{x}_{1} & 1 \\
\dot{x}_{2} & 0 & -1 & 0 & 0 & 0 \\
0 & 1 & 0 & 0 & 0 & 0
\end{array}\right) .
\end{aligned}
$$

However,

$$
\operatorname{rk}\left(\mathcal{T}_{0} \mathbf{A}(\lambda)\right)=3 \neq \operatorname{rk}\left(\overline{\mathcal{T}}_{0} \mathbf{A}(\lambda)\right)=4 .
$$

For $\beta=1$ we get

$$
\begin{aligned}
& \mathfrak{T}_{1,0}\left(\mathbf{A}_{0}\right)=\left(\begin{array}{ccc}
\mathbf{A}_{0} & \mathbf{0} & \mathbf{0} \\
\dot{\mathbf{A}}_{0} & \mathbf{A}_{0} & \mathbf{0}
\end{array}\right), \\
& \mathfrak{T}_{1,1}\left(\mathbf{A}_{1}\right)=\left(\begin{array}{ccc}
\mathbf{0} & \mathbf{A}_{1} & \mathbf{0} \\
\mathbf{0} & \dot{\mathbf{A}}_{1} & \mathbf{A}_{1}
\end{array}\right)
\end{aligned}
$$

and thus

$$
\begin{aligned}
& \mathcal{T}_{1} \mathbf{A}(\lambda) \\
= & \sum_{i=0}^{1} \mathfrak{T}_{1, i}\left(\mathbf{A}_{i}\right) \\
= & \left(\begin{array}{cccc|ccc}
\mathbf{A}_{0} & \mathbf{A}_{1} & \mathbf{0} \\
\dot{\mathbf{A}}_{0} & \mathbf{A}_{0}+\dot{\mathbf{A}}_{1} & \mathbf{A}_{1}
\end{array}\right) \\
= & \left(\begin{array}{ccc|ccc|ccc}
0 & 0 & 0 & -\dot{x}_{2} & -\dot{x}_{1} & 1 & 0 & 0 & 0 \\
\dot{x}_{2} & 0 & -1 & 0 & 0 & 0 & 0 & 0 & 0 \\
0 & 1 & 0 & 0 & 0 & 0 & 0 & 0 & 0 \\
\hline 0 & 0 & 0 & -\ddot{x}_{2} & -\ddot{x}_{1} & 0 & -\dot{x}_{2} & -\dot{x}_{1} & 1 \\
\ddot{x}_{2} & 0 & 0 & \dot{x}_{2} & 0 & -1 & 0 & 0 & 0 \\
0 & 0 & 0 & 0 & 1 & 0 & 0 & 0 & 0
\end{array}\right) .
\end{aligned}
$$

The condition

$$
\operatorname{rk}\left(\mathcal{T}_{1} \mathbf{A}(\lambda)\right)=\operatorname{rk}\left(\overline{\mathcal{T}}_{1} \mathbf{A}(\lambda)\right)=6
$$

is fulfilled and therefore $\mathbf{A}(\lambda) \in \mathcal{U}_{3}[\lambda]$. According to (44), the inverse of $\mathbf{A}(\lambda)$ results in

$$
\mathbf{A}^{-1}(\lambda)=\left(\begin{array}{ccc}
\frac{1}{\ddot{x}_{2}} & \frac{1}{\ddot{x}_{2}} \lambda & \frac{\dot{x}_{1}}{\ddot{x}_{2}} \lambda \\
0 & 0 & 1 \\
\dot{x}_{2} & -1+\frac{\dot{x}_{2}}{\ddot{x}_{2}} \lambda & \frac{\dot{x}_{1} \dot{x}_{2}}{\ddot{x}_{2}} \lambda
\end{array}\right) .
$$

Example 4. $(\alpha=2)$ Let

$$
\mathbf{A}(\lambda)=\sum_{i=0}^{2} \mathbf{A}_{i} \lambda^{i}=\left(\begin{array}{cc}
1+\lambda+\lambda^{2} & \dot{x}_{1}+x_{1} \lambda \\
x_{2}+x_{2} \lambda & x_{1} x_{2}
\end{array}\right) .
$$

For $\beta=2$ we get

$$
\begin{aligned}
\mathfrak{T}_{2,0}\left(\mathbf{A}_{0}\right) & =\left(\begin{array}{ccccc}
\mathbf{A}_{0} & \mathbf{0} & \mathbf{0} & \mathbf{0} & \mathbf{0} \\
\dot{\mathbf{A}}_{0} & \mathbf{A}_{0} & \mathbf{0} & \mathbf{0} & \mathbf{0} \\
\ddot{\mathbf{A}}_{0} & 2 \dot{\mathbf{A}}_{0} & \mathbf{A}_{0} & \mathbf{0} & \mathbf{0}
\end{array}\right), \\
\mathfrak{T}_{2,1}\left(\mathbf{A}_{1}\right) & =\left(\begin{array}{cccccc}
\mathbf{0} & \mathbf{A}_{1} & \mathbf{0} & \mathbf{0} & \mathbf{0} \\
\mathbf{0} & \dot{\mathbf{A}}_{1} & \mathbf{A}_{1} & \mathbf{0} & \mathbf{0} \\
\mathbf{0} & \ddot{\mathbf{A}}_{1} & 2 \dot{\mathbf{A}}_{1} & \mathbf{A}_{1} & \mathbf{0}
\end{array}\right), \\
\mathfrak{T}_{2,2}\left(\mathbf{A}_{2}\right) & =\left(\begin{array}{cccccc}
\mathbf{0} & \mathbf{0} & \mathbf{A}_{2} & \mathbf{0} & \mathbf{0} \\
\mathbf{0} & \mathbf{0} & \dot{\mathbf{A}}_{2} & \mathbf{A}_{2} & \mathbf{0} \\
\mathbf{0} & \mathbf{0} & \ddot{\mathbf{A}}_{2} & 2 \dot{\mathbf{A}}_{2} & \mathbf{A}_{2}
\end{array}\right) .
\end{aligned}
$$

Hence

$$
\begin{aligned}
& \mathcal{T}_{2} \mathbf{A}(\lambda) \\
& =\sum_{i=0}^{3} \mathfrak{T}_{2, i}\left(\mathbf{A}_{i}\right) \\
& =\left(\begin{array}{ccc}
\mathbf{A}_{0} & \mathbf{A}_{1} & \mathbf{A}_{2} \\
\dot{\mathbf{A}}_{0} & \mathbf{A}_{0}+\dot{\mathbf{A}}_{1} & \mathbf{A}_{1}+\dot{\mathbf{A}}_{2} \\
\ddot{\mathbf{A}}_{0} & 2 \dot{\mathbf{A}}_{0}+\ddot{\mathbf{A}}_{1} & \mathbf{A}_{0}+2 \dot{\mathbf{A}}_{1}+\ddot{\mathbf{A}}_{2}
\end{array}\right. \\
& \left.\begin{array}{cc}
\mathbf{0} & \mathbf{0} \\
\mathbf{A}_{2} & \mathbf{0} \\
+2 \dot{\mathbf{A}}_{2} & \mathbf{A}_{2}
\end{array}\right) \\
& =\left(\begin{array}{cc|cc}
1 & \dot{x}_{1} & 1 & x_{1} \\
x_{2} & x_{1} x_{2} & x_{2} & 0 \\
\hline 0 & \ddot{x}_{1} & 1 & 2 \dot{x}_{1} \\
\dot{x}_{2} & x_{1} \dot{x}_{2}+x_{2} \dot{x}_{1} & x_{2}+\dot{x}_{2} & x_{1} x_{2} \\
\hline 0 & x_{1}^{(3)} & 0 & 3 \ddot{x}_{1} \\
\ddot{x}_{2} & \star & \ddot{x}_{2}+2 \dot{x}_{2} & \dagger
\end{array}\right. \\
& \left.\begin{array}{cc|cc|cc}
1 & 0 & 0 & 0 & 0 & 0 \\
0 & 0 & 0 & 0 & 0 & 0 \\
\hline 1 & x_{1} & 1 & 0 & 0 & 0 \\
x_{2} & 0 & 0 & 0 & 0 & 0 \\
\hline 1 & 3 \dot{x}_{1} & 1 & x_{1} & 1 & 0 \\
x_{2}+2 \dot{x}_{2} & x_{1} x_{2} & x_{2} & 0 & 0 & 0
\end{array}\right)
\end{aligned}
$$

with $\star:=x_{1} \ddot{x}_{2}+x_{2} \ddot{x}_{1}+2 \dot{x}_{1} \dot{x}_{2}$ and $\dagger:=2 x_{1} \dot{x}_{2}+2 x_{2} \dot{x}_{1}$. The condition

$$
\operatorname{rk}\left(\mathcal{T}_{2} \mathbf{A}(\lambda)\right)=\operatorname{rk}\left(\overline{\mathcal{T}}_{2} \mathbf{A}(\lambda)\right)=6
$$


holds, which implies $\mathbf{A}(\lambda) \in \mathcal{U}_{2}[\lambda]$. An inverse can be determined by (44), which results in

$$
\begin{aligned}
& \mathbf{A}^{-1}(\lambda) \\
& =\left(\begin{array}{c}
1 \\
-\frac{1}{x_{1}}(1+\lambda) \\
\\
\frac{\dot{x}_{2}}{x_{2}^{2}}-\frac{1}{x_{2}} \lambda \\
\frac{x_{2}^{2}-x_{2} \ddot{x}_{2}-x_{2} \dot{x}_{2}+2 \dot{x}_{2}^{2}}{x_{1} x_{2}^{3}}+\frac{x_{2}-2 \dot{x}_{2}}{x_{1} x_{2}^{2}} \lambda+\frac{1}{x_{1} x_{2}} \lambda^{2}
\end{array}\right) .
\end{aligned}
$$

4.2. Implications. Now, we apply these results and deduce some facts about transposition of Ore polynomial matrices.

Corollary 7. Any hyper-row or hyper-column of a unimodular matrix is hyper-regular.

Proof. Assume that $\mathbf{A}(\lambda) \in \mathcal{U}_{n}[\lambda]$. This implies the existence of $\mathbf{A}^{-1}(\lambda) \in \mathcal{U}_{n}[\lambda]$ such that $\mathbf{A}(\lambda) \mathbf{A}^{-1}(\lambda)=$ $\mathbf{A}^{-1}(\lambda) \mathbf{A}(\lambda)=\mathbf{I}_{n}$. The rows of $\mathbf{A}(\lambda)$ can be re-sorted by premultiplying $\mathbf{A}(\lambda)$ by $\mathbf{R}_{\pi} \in \operatorname{Sym}(n)$ and the columns by postmultiplying by $\mathbf{C}_{\pi} \in \operatorname{Sym}(n)$, which still implies the existence of a unimodular inverse:

$$
\underbrace{\mathbf{R}_{\pi} \mathbf{A}(\lambda) \mathbf{C}_{\pi}}_{=: \tilde{\mathbf{A}}(\lambda)} \underbrace{\mathbf{C}_{\pi}^{\top} \mathbf{A}^{-1}(\lambda) \mathbf{R}_{\pi}^{\top}}_{=: \tilde{\mathbf{A}}^{-1}(\lambda)}=\mathbf{I}_{n} .
$$

Splitting $\tilde{\mathbf{A}}(\lambda) \in \mathcal{U}_{n}[\lambda]$ into hyper-rows and its inverse into hyper-columns leads to

$$
\underbrace{\left(\begin{array}{c}
\tilde{\mathbf{A}}_{1}(\lambda) \\
\tilde{\mathbf{A}}_{2}(\lambda)
\end{array}\right)}_{:=\tilde{\mathbf{A}}(\lambda)} \underbrace{\left(\tilde{\mathbf{B}}_{1}(\lambda), \tilde{\mathbf{B}}_{2}(\lambda)\right)}_{:=\tilde{\mathbf{A}}^{-1}(\lambda)}=\left(\begin{array}{ll}
\mathbf{I} & \mathbf{0} \\
\mathbf{0} & \mathbf{I}
\end{array}\right)
$$

from which we can deduce a hyper-regular right inverse of $\tilde{\mathbf{A}}_{1}(\lambda) \in \mathfrak{K}^{m \times n}[\lambda]$ to be $\tilde{\mathbf{B}}_{1}(\lambda) \in \mathfrak{K}^{n \times m}[\lambda]$, where $m<$ $n$. Similarly, splitting $\tilde{\mathbf{A}}(\lambda) \in \mathcal{U}_{n}[\lambda]$ into hyper-columns and its inverse into hyper-rows leads to

$$
\underbrace{\left(\begin{array}{c}
\tilde{\mathbf{B}}_{1}(\lambda) \\
\tilde{\mathbf{B}}_{2}(\lambda)
\end{array}\right)}_{:=\tilde{\mathbf{A}}^{-1}(\lambda)} \underbrace{\left(\tilde{\mathbf{A}}_{1}(\lambda), \tilde{\mathbf{A}}_{2}(\lambda)\right)}_{:=\tilde{\mathbf{A}}(\lambda)}=\left(\begin{array}{ll}
\mathbf{I} & \mathbf{0} \\
\mathbf{0} & \mathbf{I}
\end{array}\right)
$$

and we deduce a hyper-regular left inverse of $\tilde{\mathbf{A}}_{1}(\lambda) \in$ $\mathfrak{K}^{n \times m}[\lambda]$ to be $\tilde{\mathbf{B}}_{1}(\lambda) \in \mathfrak{K}^{m \times n}[\lambda], m<n$.

When operating with matrices whose entries are elements in $\mathfrak{K}[\lambda]$, a somewhat counterintuitive result relating to transposition can be stated as follows:

Corollary 8. The following holds:

$$
\mathbf{A}(\lambda) \in \mathcal{U}_{n}[\lambda] \Rightarrow \mathbf{A}^{\top}(\lambda) \in \mathcal{U}_{n}[\lambda] .
$$

Proof. (Counter example) Let

$$
\mathbf{A}(\lambda)=\left(\begin{array}{cc}
1+\lambda+\lambda^{2} & \dot{x}_{1}+x_{1} \lambda \\
x_{2}+x_{2} \lambda & x_{1} x_{2}
\end{array}\right) .
$$

For both, $\mathbf{A}(\lambda) \in \mathcal{U}_{2}[\lambda]$ and its transpose, the order $\beta$ is bounded by $\operatorname{deg} \mathbf{A}(\lambda) \cdot(2-1)=\operatorname{deg} \mathbf{A}(\lambda)=2$ (see Propostition 3). As shown in Example 4, $\mathbf{A}(\lambda) \in \mathcal{U}_{2}[\lambda]$, but

$$
\operatorname{rk}\left(\mathcal{T}_{\beta} \mathbf{A}^{\top}(\lambda)\right) \neq \operatorname{rk}\left(\overline{\mathcal{T}}_{\beta} \mathbf{A}^{\top}(\lambda)\right) \forall \beta \in\{0,1,2\}
$$

and therefore $\mathbf{A}^{\top}(\lambda) \notin \mathcal{U}_{2}[\lambda]$.

Corollary 9. Let $\mathbf{A}(\lambda) \in \mathfrak{K}^{m \times n}[\lambda]$. The following holds:

$$
\mathbf{A}(\lambda) \text { hyper-regular } \Longrightarrow \mathbf{A}^{\top}(\lambda) \text { hyper-regular. }
$$

Proof. This conclusion follows easily from Corollaries 7 and 8 .

These results are consistent with the fact that a division ring is a field, i.e., commutative if and only if the set of invertible matrices over the ring is closed under transposition (see Jacobson, 1953, p. 24, ex. 3; Gupta et al., 2009).

In particular, it should be noted that $\mathbf{A}(\lambda) \mathbf{R}(\lambda)=$ $\mathbf{I} \Rightarrow \mathbf{R}^{\top}(\lambda) \mathbf{A}^{\top}(\lambda)=\mathbf{I}$ for $\mathbf{A}(\lambda) \in \mathfrak{K}^{m \times n}[\lambda]$ and $\mathbf{R}(\lambda) \in \mathfrak{K}^{n \times m}[\lambda]$. Unlike Remark [3, where matrices have entries in $\mathfrak{K}$, here we operate on $\mathfrak{K}[\lambda]$, such that these implications do not violate the statement of Remark 3 .

Example 5. From

$$
\begin{aligned}
& \mathbf{A}(\lambda)=\left(\begin{array}{lll}
-\dot{x}_{2} \lambda & -\dot{x}_{1} \lambda & \lambda
\end{array}\right)
\end{aligned}
$$

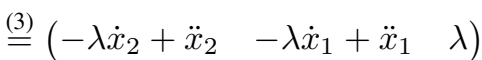

we can derive a right inverse

$$
\mathbf{R}=\left(\frac{1}{\ddot{x}_{2}}, 0, \frac{\dot{x}_{2}}{\ddot{x}_{2}}\right)^{\top}
$$

since

$$
\mathbf{A}(\lambda) \mathbf{R}=\left(\begin{array}{lll}
-\lambda \dot{x}_{2}+\ddot{x}_{2} & -\lambda \dot{x}_{1}+\ddot{x}_{1} & \lambda
\end{array}\right)\left(\begin{array}{c}
\frac{1}{\ddot{x}_{2}} \\
0 \\
\frac{\dot{x}_{2}}{\ddot{x}_{2}}
\end{array}\right)=1
$$

holds, but

$$
\begin{aligned}
\mathbf{R}^{\top} \mathbf{A}^{\top}(\lambda) & =\left(\begin{array}{lll}
\frac{1}{\ddot{x}_{2}} & 0 & \frac{\dot{x}_{2}}{\ddot{x}_{2}}
\end{array}\right)\left(\begin{array}{c}
-\dot{x}_{2} \lambda \\
-\dot{x}_{1} \lambda \\
\lambda
\end{array}\right) \\
& =-\frac{\dot{x}_{2}}{\ddot{x}_{2}} \lambda+\frac{\dot{x}_{2}}{\ddot{x}_{2}} \lambda=0 \neq 1 .
\end{aligned}
$$




\section{Conclusion and outlook}

We have investigated matrices with meromorphic entries in the differential operator $\frac{\mathrm{d}}{\mathrm{d} t}$. In particular, we have transformed the problem of proving hyper-regularity for such matrices into checking rank conditions. Despite dealing with symbolic entries, these can be evaluated very efficiently by computer algebra systems such as Maxima or toolboxes like Python's SymPy (see Remark 5 or the results of Knoll (2016, p. 176)). For this purpose, it can be shown that hyper-regularity is equivalent to right and left invertibility, respectively. Right (left) invertibility can be shown by starting with a left (right) polynomial ansatz of arbitrary degree for the inverse, left (right) shifting the differential operator and comparing the resulting coefficients, which leads to a system of linear equations. In order to avoid non-commutative shifting operations, we have introduced operators that greatly simplify the assembly of these linear equations.

Using this approach, all computations can be done commutatively in the field of meromorphic functions $\mathfrak{K}$ instead of the ring $\mathfrak{K}[\lambda]$. Examining the solvability of these equations leads to the proposed rank conditions as well as to the computation of hyper-regular and unimodular inverses. Upper degree bounds for hyper-regular and unimodular inverses are well known (Beckermann et al., 2006; Lévine, 2011; Ritt, 1935; Ollivier, 1990; Kondratieva et al., 1982; Ollivier and Brahim, 2007) and make the proposed methods practical.

The results are supported by a free and open source 4 Python toolbox (Fritzsche, 2018) based on SymPy (Meurer et al., 2017) which allows us to reproduce the examples shown in the contribution.

While computing orthogonal complements of hyper-regular matrices is possible with similar approaches, hyper-regularity of these complements cannot be ensured without additional efforts, such that the computation of unimodular completions (i.e., hyper-regular orthogonal complements of hyper-regular inverses) using the proposed methods is not fully finalized.

\section{Acknowledgment}

The authors would like to thank Carsten Knoll and the anonymous reviewers for their comments and suggestions.

\section{References}

Antritter, F., Cazaurang, F., Lévine, J. and Middeke, J. (2014). On the computation of $\pi$-flat outputs for linear time-varying differential-delay systems, Systems \& Control Letters 71: 14-22.

${ }^{4}$ GNU General Public License, Version 3.
Antritter, F. and Middeke, J. (2011). A toolbox for the analysis of linear systems with delays, Proceedings of CDC-ECC, Orlando, FL, USA, pp. 1950-1955.

Beckermann, B., Cheng, H. and Labahn, G. (2006). Fraction-free row reduction of matrices of Ore polynomials, Journal of Symbolic Computation 41(5): 513-543.

Ben-Israel, A. and Greville, T.N. (2003). Generalized Inverses: Theory and Applications, Springer, New York, NY.

Bose, N.K. and Mitra, S.K. (1978). Generalized inverse of polynomial matrices, IEEE Transactions on Automatic Control 23(3): 491-493.

Boullion, T.L. and Odell, P.L. (1971). Generalized Inverse Matrices, Wiley, New York, NY.

Campbell, S.L. and Meyer, C.D. (2008). Generalized Inverses of Linear Transformations, SIAM, London.

Cluzeau, T. and Quadrat, A. (2013). Isomorphisms and Serre's reduction of linear systems, Proceedings of the 8th International Workshop on Multidimensional Systems, Erlangen, Germany, pp. 11-16.

Cohn, P. (1985). Free Rings and Their Relations, Academic Press, London.

Davies, P., Cheng, H. and Labahn, G. (2008). Computing Popov form of general Ore polynomial matrices, Proceedings of the Conference on Milestones in Computer Algebra (MICA), Stonehaven Bay, Trinidad and Tobago, pp. $149-156$

Fabianska, A. and Quadrat, A. (2007). Applications of the Quillen-Suslin theorem to multidimensional systems theory, in H. Park and G. Regensburger (Eds.), Gröbner Bases in Control Theory and Signal Processing, De Gruyter, Berlin/New York, NY, pp. 23-106.

Franke, M. and Röbenack, K. (2013). On the computation of flat outputs for nonlinear control systems, Proceedings of the European Control Conference (ECC), Zürich, Switzerland, pp. 167-172.

Fritzsche, K. (2018). Toolbox for checking hyper regularity and unimodularity of polynomial matrices in the differential operator d/dt, https://github.com/klim-/hypo re.

Fritzsche, K., Knoll, C., Franke, M. and Röbenack, K. (2016). Unimodular completion and direct flat representation in the context of differential flatness, Proceedings in Applied Mathematics and Mechanics 16(1): 807-808.

Gupta, R.N., Khurana, A., Khurana, D. and Lam, T.Y. (2009). Rings over which the transpose of every invertible matrix is invertible, Journal of Algebra 322(5): 1627-1636.

Jacobson, N. (1953). Lectures in Abstract Algebra II: Linear Algebra, Springer, New York, NY.

Knoll, C. (2016). Regelungstheoretische Analyse- und Entwurfsansätze für unteraktuierte mechanische Systeme, $\mathrm{PhD}$ thesis, TU Dresden, Dresden.

Knoll, C. and Fritzsche, K. (2017). Symbtools: A toolbox for symbolic calculations in nonlinear control theory, DOI: 10.5281/zenodo.275073. 
Kondratieva, M.V., Mikhalev, A.V. and Pankratiev, E.V. (1982). On Jacobi's bound for systems of differential polynomials, Algebra, Moscow University Press, Moscow, pp. 79-85.

Lam, T. (1978). Serre's Conjecture, Springer, Berlin/Heidelberg.

Lévine, J. (2011). On necessary and sufficient conditions for differential flatness, Applicable Algebra in Engineering, Communication and Computing 22(1): 47-90.

Logar, A. and Sturmfels, B. (1992). Algorithms for the Quillen-Suslin theorem, Journal of Algebra 145(1): 231-239.

Meurer, A., Smith, C., Paprocki, M., Čertík, O., Kirpichev, S., Rocklin, M., Kumar, A., Ivanov, S., Moore, J., Singh, S., Rathnayake, T., Vig, S., Granger, B., Muller, R., Bonazzi, F., Gupta, H., Vats, S., Johansson, F., Pedregosa, F., Curry, M., Terrel, A., Roučka, v., Saboo, A., Fernando, I., Kulal, S., Cimrman, R. and Scopatz, A. (2017). SymPY: Symbolic computing in Python, PeerJ Computer Science 3: e103, DOI: 10.7717/peerj-cs.103.

Middeke, J. (2011). A Computational View on Normal Forms of Matrices of Ore Polynomials, $\mathrm{PhD}$ thesis, Johannes Kepler Universität Linz, Linz.

Newman, M. (1972). Integral Matrices, Academic Press, New York, NY/London.

Ollivier, F. (1990). Standard bases of differential ideals, Proceedings of the 8th International Conference on Applied Algebra, Algebraic Algorithms and Error-Correcting Codes, Tokyo, Japan, pp. 304-321.

Ollivier, F. and Brahim, S. (2007). La borne de Jacobi pour une diffiété définie par un système quasi régulier (Jacobi's bound for a diffiety defined by a quasi-regular system), Comptes rendus Mathématiques 345(3): 139-144.

Ritt, J.F. (1935). Jacobi's problem on the order of a system of differential equations, Annals of Mathematics 36(2): 303-312.
Röbenack, K. and Reinschke, K. (2011). On generalized inverses of singular matrix pencils, International Journal of Applied Mathematics and Computer Science 21(1): 161-172, DOI: 10.2478/v10006-011-0012-3.

Verhoeven, G.G. (2016). Symbolic Software Tools for Flatness of Linear Systems with Delays and Nonlinear Systems, $\mathrm{PhD}$ thesis, Universität der Bundeswehr München, Munich.

Youla, D. and Pickel, P. (1984). The Quillen-Suslin theorem and the structure of $n$-dimensional elementary polynomial matrices, IEEE Transactions on Circuits and Systems 31(6): 513-518.

Zhou, W. and Labahn, G. (2014). Unimodular completion of polynomial matrices, Proceedings of the 41 st International Symposium on Symbolic and Algebraic Computation (ISSAC), Kobe, Japan, pp. 414-420.

Klemens Fritzsche received his Dipl.-Ing. degree in mechatronics engineering from the Dresden University of Technology in 2015. He is a scientific co-worker at the Institute of Control Theory. His research interests include algebraic methods in nonlinear control, especially differential flatness.

Klaus Röbenack holds a doctoral degree in electrical engineering and a Dipl.-Math. degree from the Dresden University of Technology. His research interests include nonlinear control, observer design, descriptor systems and scientific computing. Prof. Röbenack has been the head of the Institute of Control Theory at the Dresden University of Technology since 2009.

Received: 18 August 2017 Revised: 27 February 2018 Re-revised: 16 April 2018 Accepted: 26 April 2018 